\title{
A NOTE ON CLUSTER POINTS OF A SEMIHEREDITARY STABLE SYSTEM OF SETS
}

\author{
BY W. F. PFEFFER AND W. J. WILBUR
}

1. Introduction. It has been shown in [2] and [3] that those differential properties of set functions which generalize the fundamental theorem of calculus can be studied by means of cluster points of semihereditary and stable systems of subsets of a topological space. These systems were investigated there and among other things the following was proved:

(a) Every nonempty system which is semihereditary and stable with respect to a prering has uncountably many weak cluster points ([3, Theorem 2.4]).

(b) Every nonempty system which is semihereditary and stable with respect to a prering has at least the continuum of cluster points, provided the underlying topological space is locally pseudometrizable ([3, Theorem 3.2]).

(c) Every nonempty system which is semihereditary with respect to a ring has a cluster point, provided the underlying topological space is Hausdorff and locally compact ([2, Theorem 4.3]).

It is the purpose of this paper to show that in a regular topological space every nonempty system which is semihereditary and stable with respect to a ring has at least the continuum of cluster points. This will be proved by combining the methods used in proofs of $[2$, Theorem $4.3]$ and [3, Theorem 3.2].

It seems to be very difficult to generalize our result for systems which are semihereditary and stable with respect to a prering only (even the question of the existence of a cluster point remains open here). Some contribution in this direction will be given in $\$ 4$.

2. Preliminaries. Throughout, $P$ is a regular topological space and $P^{-}=P \cup(\infty)$ is a one-point compactification of $P$. If $A \subset P^{-}$, then $A^{-}$and $A^{\circ}$ denote the closure and the interior of $A$ in $P^{-}$, respectively. For $x \in P^{-}, \Gamma_{x}$ is a local base at $x$ in $P^{-}$(see $[1$, p. 50]). By $\exp P$ we denote the collection of all subsets of $P$. If $\delta \subset \exp P$ and $A \subset P^{-}$, then $\delta_{\boldsymbol{A}}=\{B \in \delta: B \subset A\}$. Finally, we shall choose a fixed system $\sigma \subset \exp P$ such that $\Gamma_{x} \subset \sigma$ for every $x \in P$. The following assumptions about the system $\sigma$ will be used:

(i) If $A, B \in \sigma$, then $A-B \in \sigma$.

Received by the editors February 27, 1968. 
(ii) If $A, B \in \sigma$, then $A \cap B \in \sigma$ and $A-B=\bigcup_{i=1}^{n} C_{i}$ where $C_{1}, \cdots$, $C_{n}$ are disjoint sets from $\sigma$.

A system $\sigma$ which satisfies condition (i) or (ii) is called a difference system or a prering, respectively. Obviously, every ring is a difference system and every difference system is a prering; for $A \cap B=A$ $-(A-B)$.

2.1. Definition. A system $\delta \subset \sigma$ is said to be semihereditary if and only if $\sigma_{0} \cap \delta \neq \varnothing$ for every finite disjoint collection $\sigma_{0} \subset \sigma$ for which $\bigcup\left\{A: A \in \sigma_{0}\right\} \in \delta$.

By a simple induction it follows that if $\sigma$ is a difference system, then $\delta \subset \sigma$ is semihereditary whenever for every $A \in \delta$ and every $B \in \sigma$ either $A \cap B \in \delta$ or $A-B \in \delta$. This fact relates our definition of semihereditariness to the definition given in [2, Definition 3.2].

2.2. Definition. A system $\delta \subset \exp P$ is said to be stable if and only if $\varnothing \notin \delta$ and for every $A \in \delta$ and every $x \in P^{-}$there is a $U \in \Gamma_{x}$ such that $\delta_{A-U} \neq \varnothing$.

2.3. Definition. Let $\delta \subset \exp P$. A point $x \in P^{-}$is said to be a cluster point of $\delta$ if and only if for every $U \in \Gamma_{x}$ there is an $A \in \delta_{U}$ such that $x \in A^{-}$.

The set of all cluster points of $\delta$ is denoted by $\delta^{c}$.

For a motivation of Definitions $2.1-2.3$ see $[3, \S 4]$.

3. Main theorem. From now on $\delta \subset \sigma$ is a given system which does not contain the empty set and $\Delta$ is the family of all nonempty compact sets $C \subset P^{-}$such that for every open set $G \subset P^{-}$containing $C$ there is a set $A \in \delta$ for which $C \subset A^{-} \subset G$. Notice that if $A \in \delta$, then $A^{-} \in \Delta$.

\subsection{Lemma. If $\Delta_{0} \subset \Delta$ is a nonempty chain, ${ }^{1}$ then $\cap \Delta_{0} \in \Delta$.}

Proof. Obviously, $\cap \Delta_{0}$ is nonempty and compact. Let $G \subset P^{-}$be an open set containing $\cap \Delta_{0}$. Then $\cap\left\{C-G: C \in \Delta_{0}\right\}=\cap \Delta_{0}-G=\varnothing$. Because $\left\{C-G: C \in \Delta_{0}\right\}$ is a chain of compact sets, $C \subset G$ for some $C \in \Delta_{0}$ and the lemma follows.

3.2. Corollary. If $C \in \Delta$ then there is a set $C_{0} \in \Delta_{C}$ which is minimal in $\Delta$.

The corollary follows from [1, p. 33, Theorem 25(b)].

3.3. Lemma. Let $\sigma$ be a difference system. If $\delta$ is semihereditary then every minimal set in $\Delta$ is a singleton.

Proof. Let $D$ be a minimal set in $\Delta$. If $D \subset\{x\}-$ for some $x \in P^{-}$, then it follows immediately from the regularity of $P$ that $D$ is a sin-

\footnotetext{
${ }^{1}$ See $[1$, p. 32].
} 
gleton. Hence suppose that $D$ contains two points $x$ and $y$ for which $\{x\}-\cap\{y\}-=\varnothing$ and $x \neq \infty$. Since $\{x\} \notin \Delta$ there is an open set $G \subset P^{-}$ such that $x \in G$ and for no set $A \in \delta$ is $x \in A^{-} \subset G$. The point $x$ has a neighborhood $U \in \sigma$ for which $U \subset G-\{y\}$. So $D-U^{\circ}$ is a nonempty compact proper subset of $D$. Let $E \subset P^{-}$be an open set containing $D-U^{\circ}$. Without loss of generality we may assume that $x \notin E$. Because $D-E$ is a compact subset of $U^{\circ}$, it follows from [2, Proposition 4.1] that we can find a $V \in \sigma$ such that $D-E \subset V^{\circ} \subset V^{-} \subset U^{\circ}$. Hence $E \cup V^{\circ}$ is an open set containing $D$ and so there is an $A \in \delta$ for which $D \subset A^{-} \subset E \cup V^{\circ}$. Because $x \in A^{-}$and $x \in V^{\circ}$, we have $x \in(A \cap V)^{-}$ $C G$. By the choice of $G$ and the semihereditariness of $\delta, A-V \in \delta$. Since

$$
D-U^{\circ} \subset A^{-}-V^{-} \subset(A-V)^{-} \subset\left(A-V^{\circ}\right)^{-} \subset A^{-}-V^{\circ} \subset E,
$$

it follows that $D-U^{\circ} \in \Delta$. This contradicts the minimality of $D$ in $\Delta$.

3.4. Proposition. Let every minimal set in $\Delta$ be a singleton. If $\delta$ is nonempty so is $\delta^{c}$, and if, in addition, $\delta$ is also stable, then the cardinality of $\delta^{c}$ is not less than the continuum.

Proof. By the definition of $\Delta, x \in \delta^{c}$ whenever $\{x\} \in \Delta$. Hence the first part of the proposition follows directly from Corollary 3.2 and for the second part it will suffice to show that $\Delta$ contains at least the continuum of singletons. Using the stability of $\delta$ we can find a set $A \in \delta$ for which $A^{-} \subset P$. By Corollary 3.2 there is a singleton $\left\{x_{0}\right\}$ $\in \Delta_{A^{-}}$. Again from the stability of $\delta$ and Corollary 3.2 follows the existence of a set $B \in \delta_{A}$ for which $x_{0} \in B^{-}$and of a singleton $\left\{x_{1}\right\}$ $\in \Delta_{B^{-}}$. By the definition of $\Delta$ and the regularity of $P$ there are sets $A_{0}, A_{1}$ from $\delta_{A}$ for which $A_{0}^{-} \cap A_{1}^{-}=\varnothing$. By a simple induction we can define sets $A_{i_{1} \cdots i_{n}} \in \delta_{A}, n=1,2, \cdots$, such that $A_{i_{1} \cdots i_{n-1} i_{n}} \subset A_{i_{1} \cdots i_{n-1}}$ and $A_{i_{1} \ldots i_{n-1} 0}^{-} \cap A_{i_{1} \ldots i_{n-1} 1}^{-}=\varnothing$, for $i_{j}=0,1, j=1,2, \cdots, n$. According to Lemma 2.1 the sets $\bigcap_{n=1}^{\infty} A_{i_{1} \ldots i_{n}}^{-}$belong to $\Delta$. Using the axiom of choice and Corollary 3.2 we associate with every sequence $\left\{i_{j}\right\}_{j=1}^{\infty}$ of zeros and ones a point $x_{i_{1} i_{2}} \ldots \in \bigcap_{n=1}^{\infty} A_{i_{1} \ldots i_{n}}^{-}$for which $\left\{x_{i_{1} i_{2}} \ldots\right\} \in \Delta$. Since different points are associated with different sequences, the proposition follows.

Combining Proposition 3.4 with Lemma 3.3 we obtain the following theorem.

3.5. THEOREM. Let $\sigma$ be a difference system and let $\delta$ be semihereditary. If $\delta$ is nonempty so is $\delta^{c}$, and if, in addition, $\delta$ is also stable, then the cardinality of $\delta^{c}$ is not less than the continuum.

4. Some results concerning prerings. Throughout this section we shall assume that in addition to regularity $P$ is also locally compact, 
and that $\sigma$ is a prering. From the local compactness of $P$ follows the regularity of $P^{-}$. The symbols $\delta$ and $\Delta$ will have the same meaning as in $\S 3$.

4.1. Proposition. If $\delta$ is nonempty, nonstable, and semihereditary, then $\delta^{c}$ is nonempty.

Proof. Since $\delta$ is nonempty and nonstable, there is a set $A \in \delta$ and a point $x \in P^{-}$such that $\delta_{A-U}=\varnothing$ for all $U \in \Gamma_{x}$. Clearly $x \in A^{-}$and we shall show that $x \in \delta^{c}$. First assume that there is a set $B \in \delta_{A}$ for which $B^{-} \subset P$. It follows that $x \neq \infty$. For a given $U \in \Gamma_{x}, A$ $=(A \cap U) \cup \cup_{i=1}^{n} B_{i}$, where $B_{1}, \cdots, B_{n}$ are disjoint sets from $\sigma_{A-U}$. Thus by the semihereditariness of $\delta, A \cap U \in \delta$ and therefore $x \in \delta^{c}$. Now let $\infty \in B^{-}$for every $B \in \delta_{A}$. It follows from the semihereditariness of $\delta$ and the regularity of $P^{-}$that $x=\infty$. Given $U \in \Gamma_{x}$ we can find a $V \in \Gamma_{x}$ such that $V^{-} \subset U^{\circ}$. Since the sets $U^{\circ}$ and $P^{-}-V^{-}$form an open cover of $A$ we see from [3, (1.3)] that there are disjoint sets $C_{i} \in \sigma_{A}$ such that either $C_{i} \subset U^{\circ}$ or $C_{i} \subset P^{-}-V^{-}, i=1,2, \cdots, m$, and $\bigcup_{i=1}^{n} C_{i}=A$. By the semihereditariness of $\delta$ at least one of the sets $C_{i}$, say $C_{1}$, belongs to $\delta$. According to our assumption $C_{1} \subset U$ (for $\infty \in C_{1}^{-}$) and hence again $x \in \delta^{c}$.

In view of the previous proposition it remains to study only those semihereditary systems all semihereditary subsystems of which are stable. The following example shows that such systems actually exist.

4.2. ExAmple. Let $P=[0, \sqrt{2})$ together with the ordinary topology, let $\sigma$ be a prering consisting of all half-open intervals $[a, b) \subset P$, and let $\delta$ be the system consisting of those intervals $[a, b) \in \sigma$ for which $b-a$ is irrational. We shall identify $P^{-}$with $[0, \sqrt{2}]$. If $[a, b) \in \delta$ and $[a, b)=\bigcup_{i=1}^{n}\left[a_{i}, b_{i}\right)$ where the union is disjoint, then $b-a=\sum_{n=1}^{n}\left(b_{i}-a_{i}\right)$. Since $b-a$ is irrational, so is $b_{i}-a_{i}$ for some $i, 1 \leqq i \leqq n$, and the semihereditariness of the system $\delta$ follows. Let $\delta^{\prime} \subset \delta$ be semihereditary, $[a, b) \in \delta^{\prime}$, and $x \in P^{-}$. If $x \in(a, b)$ we can choose $[c, d] \subset(a, b)$ such that $x \in(c, d)$ and $d-c$ is rational. By the semihereditariness of $\delta^{\prime}$, either $[a, c)$ or $[d, b)$ belongs to $\delta^{\prime}$ and hence $\delta_{[a, b)-(c, d)}^{\prime} \neq 0$. Since the cases $x=a, x=b$, or $x \notin[a, b]$, can be treated similarly, we see that $\delta^{\prime}$ is stable. It should be mentioned that there exist nontrivial semihereditary subsystems of $\delta$; e.g. with the aid of Zorn's lemma it can be rather easily shown that there is a system $\delta_{0} \subset \delta$ which is a minimal semihereditary subsystem of $\delta$ containing $P$. Of course, since $P$ is metrizable, it follows from [3, Theorem 3.2] that all these systems have the continuum of cluster points.

Note. There is no superadditive function $F$ on $\sigma$ (see [3, Definition 4.1]) for which $L_{c}(F, x) \geqq 0$ for all $x \in P^{-}$(see [3, Definition 4.3]) and 
$\{A \in \sigma: F(A)<0\}=\delta$. Indeed, let $F$ be a superadditive function on $\sigma$ for which $\{A \in \sigma: F(A)<0\}=\delta$ and let $x$ be any point in $P^{-}$. We can find sequences $\left\{a_{n}\right\},\left\{b_{n}\right\}$ such that $0 \leqq a_{n} \leqq x \leqq b_{n} \leqq \sqrt{2}, a_{n}$ and $\sqrt{2}-b_{n}$ are rational, $n=1,2, \cdots$, and $\lim a_{n}=\lim b_{n}=x$. Then it follows from the superadditivity of $F$ that $F\left(\left[a_{n}, b_{n}\right)\right) \leqq F([0, \sqrt{2}))<0$ for all $n$ and hence $L_{c}(F, x)<0$. This answers negatively the question given in $[3, \$ 4.6]$.

We denote by $\sigma^{\wedge}$ the system of all sets from $\sigma$ which are both compact and open. If the topology of $P$ has a base consisting of compactopen sets ${ }^{2}$ the system $\sigma^{\wedge}$ can be quite large.

4.3. Proposition. Let $\sigma^{\wedge}$ be a prering which contains $\Gamma_{z}$ for every $x \in P$ and let $\delta$ be semihereditary. If $\delta$ is nonempty so is $\delta^{c}$, and if, in addition, $\delta$ is also stable, then the cardinality of $\delta^{c}$ is not less than the continuum.

Proof. According to Proposition 3.4 it suffices to prove that every minimal set in $\Delta$ is a singleton. Hence let $D$ be a minimal set in $\Delta$. If $D \subset\{x\}$ - for some $x \in P^{-}$, then it follows immediately from the regularity of $P^{-}$that $D$ is a singleton. Thus suppose that $D$ contains two points $x$ and $y$ for which $\{x\}-\cap\{y\}^{-}=\varnothing$. Since $D$ is compact, $D$ can be covered by finitely many disjoint sets $U_{1}, \cdots, U_{n}$ from $\sigma^{\wedge}$ (see $[3,(1.1)]$ ). Because $P^{-}$is regular we may assume that $x$ and $y$ do not belong to the same set $U_{i}, 1 \leqq i \leqq n$. Therefore, we may assume that $D_{i}=D \cap U_{i} \neq \varnothing$ for $i=1,2, \cdots, n$ and that $n \geqq 2$. It follows that $D_{1}, \cdots, D_{n}$ do not belong to $\Delta$. Since they are compact we can find open sets $V_{i}$ such that $D_{i} \subset V_{i} \subset U_{i}$ and for no set $B \in \delta$ and no $i, 1 \leqq i \leqq n$, is $D_{i} \subset B^{-} \subset V_{i}, i=1,2, \cdots, n$. On the other hand there is a set $A \in \delta$ for which $D \subset A-\subset \bigcup_{i=1}^{n} V_{i}$. If we set $A_{i}=A \cap U_{i}$ it follows from the semihereditariness of $\delta$ that for some $i_{0}, 1 \leqq i_{0} \leqq n$, $A_{i_{0}} \in \delta$. This is a contradiction, for $D_{i_{0}} \subset A^{-} \cap U_{i_{0}}=A_{i_{0}}^{-} \subset V_{i_{0}}$.

In particular, the previous proposition applies when $P$ is the Tychonoff product of any family of finite discrete spaces and $\sigma$ is the prering consisting of all rectangles which form the standard base for the topology in $P$ (in this case $\sigma^{\wedge}=\sigma$ ).

\section{REFERENCES}

1. J. L. Kelley, General topology, Van Nostrand, New York, 1955.

2. W. F. Pfeffer, $A$ note on the lower derivate of a set function and semihereditary systems of sets, Proc. Amer. Math. Soc. 18 (1967), 1020-1025.

3. - On the lower derivate of a set function, Canad. J. Math. (to appear).

University of California, Davis

${ }^{2} \mathrm{~A}$ Hausdorff space with this property is called a Boolean space (see [1, p. 168]). 